\title{
NEW DEFORMATIONS OF GROUP ALGEBRAS OF COXETER GROUPS, II
}

\author{
PAVEL ETINGOF AND ERIC RAINS
}

\begin{abstract}
Dedicated to the 60-th birthday of Joseph Bernstein
\end{abstract}
\section{INTRODUCTION}

This paper is a sequel of [ER. Specifically, let $W$ be a Coxeter group, generated by $s_{i}, i \in I$. Then, following [ER], one can define a new deformation $A_{+}=A_{+}(W)$ of the group algebra $\mathbb{Z}\left[W_{+}\right]$of the group $W_{+}$of even elements in $W$. This deformation is an algebra over the ring $R=\mathbb{Z}\left[t_{i j k}^{ \pm 1}\right]=\mathbb{Z}[\mathbb{T}]$ of regular functions on a certain torus $\mathbb{T}$ of deformation parameters. The main result of [ER implies that this deformation is flat (i.e., $A_{+}$is a flat $R$-module) if and only if for every triple of indices $\Delta=\{i, j, k\} \subset I$ the corresponding rank 3 parabolic subgroup $W_{\Delta} \subset W$ is infinite. ${ }^{1}$

Unfortunately, this result is not entirely satisfactory, since the most interesting Coxeter groups (finite, affine, hyperbolic) typically do not satisfy the condition that all the rank 3 parabolic subgroups are infinite. For this reason, it is interesting to generalize the theory of [ER] to the case of general Coxeter groups. Such a generalization is the main goal of this paper.

More precisely, recall that in ER] we constructed elements $T_{w(x)}$, labeled by $x \in W_{+}$, which span $A_{+}$over $R$. Let $J \subset R$ be the intersection of all ideals $I$ in $R$ such that $T_{w(x)}$ are a basis of $A_{+} / I A_{+}$over $R / I$, and let $\mathbb{T}_{f}=\operatorname{Spec} R / J$. If $k$ is a field, then $\mathbb{T}_{f}(k)$ is the subset of points $u \in \mathbb{T}(k)$ where the fiber $A_{u+}$ of the algebra $A_{+}$is "a flat deformation of $\mathbb{Z}\left[W_{+}\right]$" (in the sense that the spanning elements $T_{w(x)} \in A_{u+}$ are actually a basis). Thus we call $\mathbb{T}_{f}$ the flatness locus. The main result of the paper is an explicit characterization of the flatness locus. Namely, for every triple of indices $\Delta=\{i, j, k\} \subset I$ denote by $\mathbb{T}_{f}^{\Delta}$ the flatness locus for the "rank 3 subalgebra" $A_{+}^{\Delta}$ of $A_{+}$generated by $a_{i j}, a_{j k}, a_{k i}$. Note that if $W_{\Delta}$ is infinite, then $\mathbb{T}_{f}^{\Delta}=\mathbb{T}$.

It is clear that $\mathbb{T}_{f} \subset \cap_{\Delta \subset I} \mathbb{T}_{f}^{\Delta}$. One of our main results is the following theorem.

Theorem 1.1. One has $\mathbb{T}_{f}=\cap_{\Delta \subset I} \mathbb{T}_{f}^{\Delta}$.

In other words, the algebra $A_{+}$is flat at $u$ if and only if so is $A_{+}^{\Delta}$ for every $\Delta$. Obviously, this is a generalization of the main result of [ER].

\footnotetext{
${ }^{1}$ To be more precise, in $[\mathrm{ER}$ we work over $\mathbb{C}$, but the results routinely extend to the case of ground ring $\mathbb{Z}$.
} 
Theorem 1.1 reduces the problem of explicit description of the flatness locus $\mathbb{T}_{f}$ for any Coxeter group $W$ to the same problem for finite Coxeter groups of rank 3 , i.e. $A_{1} \times I_{2 m}, A_{3}, B_{3}$, and $H_{3}$. The even subgroups of these groups have a presentation by three generators $x, y, z$ of specified orders, with the additional relation that the product of the generators is 1 . Therefore, the problem of finding $\mathbb{T}_{f}$ for such groups reduces to the so called multiplicative Deligne-Simpson problem, which is the problem of existence of a collection of matrices with given spectra whose product is the identity matrix. Thus, using known results about the Deligne-Simpson problem, we obtain an explicit description of $\mathbb{T}_{f}$ in the rank 3 case.

Our description of $\mathbb{T}_{f}$ implies that $\mathbb{T}_{f}$ is a union of affine subtori of $\mathbb{T}$ (possibly of different dimensions). These tori are not disjoint, however, and are quite large in number, since they break the symmetry of the action of a large product of symmetric groups on $\mathbb{T}_{f}$. This suggests looking at the quotient of $\mathbb{T}_{f}$ by this permutation action, which has a nicer geometric structure. Namely, over $\mathbb{C}$, this quotient is the total space of a vector bundle (with dimension depending on the component) over a commutative affine algebraic group, the zero section of which parametrizes points $u$ such that the algebra $A_{u+}$ is a twisted group algebra of $W_{+}$. Over $\mathbb{Z}$, this structure nearly carries over; it is still a scheme over the locus of twisted group algebras cut out by linear equations (so the fibers are affine spaces), but can fail to be locally trivial near a nonreduced point of the base. (Note that the base is nonreduced in characteristic $\mathrm{p}$ if and only if $\mathrm{p}$ divides the number of components of $H^{*}\left(W_{+}, \mathbb{C}^{*}\right)$.)

In particular, the point $1 \in \mathbb{T}$ (at which all the generators $a_{i j}$ of $A_{+}$are unipotent) belongs to $\mathbb{T}_{f}$; the 2-cocycle $\psi$ corresponding to this point is the 2 -cocycle $\psi_{\text {spin }}$ of the spinor representation of $W_{+}$.

Note that a special case of Theorem 1.1] and the explicit description of 2-cocycles in the rank 3 case yield an explicit description of $H^{2}\left(W_{+}, \mathbb{C}^{*}\right)$. This description (which is an important ingredient in the proof of Theorem 1.1) was actually known before, and is contained in the paper $\mathrm{Bu}$.

At the end of the paper, we show that the classical Iwahori-Hecke algebra is a special case of our deformation. We also consider the additive versions $\mathcal{A}_{u+}$ of the algebras $A_{u+}$, and study their properties. In particular, we show that the Hilbert series of $\mathcal{A}_{0+}$ is $h(z) /(1+z)$, where $h(z)$ is the growth series of the Coxeter group $W$.

Acknowledgments. It is our pleasure to dedicate this paper to Joseph Bernstein. His work, as well as style of doing and explaining mathematics, have been an inspiration for generations of mathematicians.

The work of P.E. was partially supported by the NSF grant DMS-0504847 and the CRDF grant RM1-2545-MO-03. E.R. was supported in part by NSF grant DMS-0401387. The authors would also like to acknowledge that at many stages of this work they used the MAGMA package for algebraic computations $\mathrm{Ma}$. 


\section{Preliminaries}

2.1. Coxeter groups. Let $I$ be a finite set, and let $\mathbb{Z}_{\geq 2}$ denote the set of integers which are $\geq 2$. A Coxeter matrix over $I$ is a collection $M$ of elements $m_{i j} \in \mathbb{Z}_{\geq 2} \cup\{\infty\}, i, j \in I, i \neq j$, such that $m_{i j}=m_{j i}$. The rank $r$ of $M$ is, by definition, the cardinality of the set $I$.

Let $M$ be a Coxeter matrix. Then one defines the Coxeter group $W(M)$ by generators $s_{i}, i \in I$, and defining relations

$$
s_{i}^{2}=1,\left(s_{i} s_{j}\right)^{m_{i j}}=1 \text { if } m_{i j}<\infty .
$$

For brevity, from now on we will not write the dependence on $M$ explicitly, assuming that $M$ has been fixed (unless confusion is possible).

The group $W$ has a sign character $\xi: W \rightarrow\{ \pm 1\}$ given by $\xi\left(s_{i}\right)=-1$. Denote by $W_{+}$the kernel of $\xi$, i.e. the subgroup of even elements.

Let $J \subset I$ be a subset. Let $W_{J}$ be the subgroup of $W$ generated by $s_{i}, i \in J$. Then $W_{J}$ is a Coxeter group, which is called the parabolic subgroup of $W$ corresponding to $J$.

Let $V$ be the reflection representation of $W$, i.e. the space $\mathbb{C}^{r}$ with the action of $W$ given by $s_{i}\left(e_{j}\right)=e_{j}+2 \cos \left(\pi / m_{i j}\right) e_{i}, s_{i}\left(e_{i}\right)=-e_{i}$. This action $\rho: W \rightarrow G L(V)$ preserves the (possibly degenerate) inner product on $V$ given by $\left(e_{i}, e_{i}\right)=1,\left(e_{i}, e_{j}\right)=-\cos \left(\pi / m_{i j}\right)$. (here by definition if $m_{i j}=\infty$ then $\left.\pi / m_{i j}=0\right)$. Thus $\rho(W) \subset O(V), \rho\left(W_{+}\right) \subset S O(V)$.

For any $x \in W(M)$, let $l(x)$ be the length of $x$. Let $b_{n}$ be the number of elements of $W(M)$ with $l(x)=n$. The series $h_{M}(z):=\sum_{n>0} b_{n} z^{n}$ is called the growth series of $W$. It is known to be a rational function and can be computed explicitly $(\underline{B}])$.

2.2. The algebras $A, A_{+}, A_{u}, A_{u+}$. Recall the definition of the algebras $A(M), A_{+}(M)$ from [ER].

Let $t_{i j k}$ be commuting variables defined for $i \neq j$ such that $m_{i j}<\infty$, and $k \in \mathbb{Z}_{m_{i j}}$ (an integer modulo $m_{i j}$ ), so that $t_{i j k}=t_{j i,-k}^{-1}$. Let $R=\mathbb{Z}\left[t_{i j k}\right]$ be the ring of polynomials of these variables. (In ER, we took coefficients in $\mathbb{C}$, but for all of the results cited in this section, the same proof works over $\mathbb{Z}$.) Define the algebra $A$ generated over $R$ by generators $s_{i}$ with defining relations

$$
\begin{gathered}
s_{i}^{2}=1, \prod_{k=1}^{m_{i j}}\left(s_{i} s_{j}-t_{i j k}\right)=0 \text { if } m_{i j}<\infty, \\
s_{p} t_{i j k}=t_{j i k} s_{p} .
\end{gathered}
$$

Let $A_{+}$be the subalgebra of $A$ generated over $R$ by $a_{i j}:=s_{i} s_{j}$. The defining relations for $A_{+}$are

$$
\begin{gathered}
\prod_{k=1}^{m_{i j}}\left(a_{i j}-t_{i j k}\right)=0 \text { if } m_{i j}<\infty, \\
\quad a_{i j} a_{j i}=1, a_{i j} a_{j p} a_{p i}=1 .
\end{gathered}
$$

Note that $R$ is central in $A_{+}$(but not in $A$ ). 
Define the algebraic torus $\mathbb{T}=\operatorname{Spec} R$. The group $\mathbb{Z}_{2}$ acts on $\mathbb{T}$ via $t_{i j k} \rightarrow t_{j i k}$. If $u \in \mathbb{T}$ is a point over some field, then we denote by $A_{u}, A_{u+}$ the fibers of $A, A_{+}$at $u$. That is, $A_{u}=A / J_{u} A, A_{u+}=A_{+} / J_{u} A_{+}$, where $J_{u} \subset R$ is the maximal ideal corresponding to $u$. Note that $A_{u+}$ is an algebra, while $A_{u}$ is an algebra for any $u$ that is fixed under $\mathbb{Z}_{2}$. In particular, we can define the "unipotent" algebras $A_{1}, A_{1+}$, where $1 \in \mathbb{T}$ is the unit, i.e., the point where $t_{i j k}=1$ for all $i, j, k$. Explicitly, the algebra $A_{1}$ is generated by $s_{i}$ with defining relations

$$
s_{i}^{2}=1,\left(s_{i} s_{j}-1\right)^{m_{i j}}=0\left(m_{i j}<\infty\right)
$$

and $A_{1+}$ is generated by $a_{i j}=s_{i} s_{j}$, with defining relations

$$
a_{i j} a_{j i}=1, a_{i j} a_{j k} a_{k i}=1,\left(a_{i j}-1\right)^{m_{i j}}=0\left(m_{i j}<\infty\right) .
$$

Consider the subgroup $Z \subset \mathbb{G}_{m}^{r(r-1) / 2}$ of collections

$$
\left\{z_{i j} \in \mathbb{G}_{m}, i \neq j \mid z_{i j} z_{j i}=1, z_{i j} z_{j k} z_{k i}=1\right\} \cong \mathbb{G}_{m}^{r-1} .
$$

It acts on the algebra $A_{+}$by rescalings: $a_{i j} \rightarrow z_{i j} a_{i j}, t_{i j k} \rightarrow z_{i j} t_{i j k}$. Thus the algebras $A_{u+}$ are, in effect, parametrized by the quotient group $\mathbb{T} / Z$ (in the sense that $A_{u+}$ and $A_{z u+}$ are naturally isomorphic for $z \in Z$ ).

Let $x \rightarrow w(x)$ be a function assigning to every element $x \in W$ a reduced word representing $x$. Also, for any word $w$ in letters $s_{i}$, let $T_{w}$ be the corresponding element of $A$ (or $A_{u}$ ). Recall from ER that $T_{w(x)}, x \in W$, span $A$ as a left $R$-module, and hence span $A_{u}$ for each $u$ (and similarly, $T_{w(x)}, x \in W_{+}$, span $A_{+}$and $\left.A_{u+}\right)$. Let $J \subset R$ be the intersection of all ideals $I$ in $R$ such that $T_{w(x)}$ are a basis of $A_{+} / I A_{+}$over $R / I$, and let $\mathbb{T}_{f}=\operatorname{Spec} R / J$. (It is easy to see (cf. [ER]) that this property of $I$ is independent of the function $w(x)$ ). Thus $\mathbb{T}_{f} \subset \mathbb{T}$ is a closed subscheme of $\mathbb{T}$, which we call the flatness locus. Note that for any field $k, \mathbb{T}_{f}(k)$ is the set of all $u \in \mathbb{T}(k)$ such that the elements $T_{w(x)}, x \in W$ are a basis of $A_{u}$ (equivalently $T_{w(x)}, x \in W_{+}$, are a basis of $A_{u+}$ ).

Since $A_{u+}$ is unchanged if we reorder the $t_{i j k}$ for each $i<j$ (and reorder $t_{j i k}$ accordingly), we find that $\mathbb{T}_{f}$ is preserved by the natural action of the group $\prod_{i<j} S_{m_{i j}}$ on $\mathbb{T}$ (the product is taken over those $i, j$ for which $m_{i j}<$ $\infty)$. The quotient $\mathbb{T} / \prod_{i<j} S_{m_{i j}}$ is the spectrum of the ring

$$
\tilde{R}:=\mathbb{Z}\left[e_{i j}^{(k)}\left(1 \leq k \leq m_{i j}\right), 1 / e_{i j}^{\left(m_{i j}\right)}\right],
$$

where

$$
e_{j i}^{(k)}=e_{i j}^{\left(m_{i j}-k\right)} / e_{i j}^{\left(m_{i j}\right)}, e_{j i}^{\left(m_{i j}\right)}=1 / e_{i j}^{\left(m_{i j}\right)} .
$$

This gives rise to an algebra $\tilde{A}$ with presentation

$$
s_{i}^{2}=1,\left(s_{i} s_{j}\right)^{m_{i j}}+\sum_{k=1}^{m_{i j}}(-1)^{k} e_{i j}^{(k)}\left(s_{i} s_{j}\right)^{m_{i j}-k}=0 \text { if } m_{i j}<\infty, s_{p} e_{i j}^{(k)}=e_{j i}^{(k)} s_{p},
$$


and a subalgebra $\tilde{A}_{+}$with presentation

$$
a_{i j}^{m_{i j}}+\sum_{k=1}^{m_{i j}}(-1)^{k} e_{i j}^{(k)} a_{i j}^{m_{i j}-k}=0 \text { if } m_{i j}<\infty, a_{i j} a_{j i}=1, a_{i j} a_{j p} a_{p i}=1 .
$$

Denote by $\tilde{A}_{v}, \tilde{A}_{v+}$ the fiber of $\tilde{A}, \tilde{A}_{+}$, respectively, at the point $v \in$ $\mathbb{T} / \prod_{i<j} S_{m_{i j}}$. Let $\pi: \mathbb{T} \rightarrow \mathbb{T} / \prod_{i<j} S_{m_{i j}}$ be the natural projection. Note that $A_{u+} \cong \tilde{A}_{\pi(u)+}$ and $A_{u} \cong \tilde{A}_{\pi(u)}$, and thus $A_{u}$ has a natural algebra structure not only when $u$ is $\mathbb{Z}_{2}$-invariant, but also in the more general case when only $\pi(u)$ is $\mathbb{Z}_{2}$-invariant. In any event, we observe that the flatness locus associated to $\tilde{A}$ is $\tilde{\mathbb{T}}_{f}:=\pi\left(\mathbb{T}_{f}\right)$, since the coefficients of the relations of $A_{u+}$ are functions on $\tilde{T}$; in particular, this holds scheme-theoretically, not just point-wise.

\section{SCHUR MULTIPLIERS}

Let $\mathbb{T}_{f}^{S}$ be the set of all complex points of $u \in \mathbb{T}_{f}$ for which

$$
\prod_{k}\left(z-t_{i j k}\right)=z^{m_{i j}}+(-1)^{m_{i j}} t_{i j}
$$

for some complex numbers $t_{i j}\left(t_{i j}=t_{j i}^{-1}\right)$, defined when $m_{i j}<\infty$. Denote the set of the corresponding collections of numbers $t_{i j}$ by $\Theta$ (clearly, the isomorphism class of $A_{u+}, u \in \mathbb{T}_{f}^{S}$, depends only on the image of $u$ in $\Theta$, which we denote by $t=t_{u}$ ). Then the polynomial relations for $A_{u+}$ take the form $a_{i j}^{m_{i j}}=(-1)^{m_{i j}+1} t_{i j}$. Let $[x]:=T_{w(x)}$ Then in the algebra $A_{u+}$ we have the following multiplication table: $[x][y]=\psi_{t}(x, y)[x y]$, where $\psi_{t}(x, y)$ are some scalars. Indeed, the scalar $\psi_{t}(x, y)$ is \pm a product of $t_{i j}$ which appears when we express $w(x y)$ via $w(x) w(y)$ using the braid relations. Note that the braid relations written in terms of $a_{i j} \in A_{u+}$ look like

$$
\begin{aligned}
a_{i j}^{m_{i j} / 2} & =-t_{i j} a_{j i}^{m_{i j} / 2} \\
a_{p j} a_{i j}^{\left(m_{i j}-2\right) / 2} a_{i q} & =-t_{i j} a_{p i} a_{j i}^{\left(m_{i j}-2\right) / 2} a_{j q}
\end{aligned}
$$

if $m_{i j}$ is even, and

$$
\begin{aligned}
& a_{i j}^{\left(m_{i j}-1\right) / 2} a_{i p}=t_{i j} a_{j i}^{\left(m_{i j}-1\right) / 2} a_{j p}, \\
& a_{p i} a_{i j}^{\left(m_{i j}-1\right) / 2}=t_{i j} a_{p j} a_{j i}^{\left(m_{i j}-1\right) / 2}
\end{aligned}
$$

if $m_{i j}$ is odd.

Thus $\psi_{t}(x, y)$ is a Schur multiplier (a 2-cocycle of $W_{+}$with coefficients in $\left.\mathbb{C}^{*}\right)$, and $A_{u+}$ is the twisted group algebra $\mathbb{C}_{\psi_{t}}\left[W_{+}\right]$. It is obvious that if the function $w(x)$ is changed, then the 2-cocycle $\psi_{t}$ changes by a coboundary. Thus we have a canonical map $\psi: \Theta \rightarrow H^{2}\left(W_{+}, \mathbb{C}^{*}\right)$ defined by $t \rightarrow \psi_{t}$. 
Proposition 3.1. A system of numbers $t_{i j}$ is in $\Theta$ if and only if whenever $W_{i j k}$ is finite,

$$
t_{i j}^{\left[W_{i j k}: W_{i j}\right]} t_{j k}^{\left[W_{i j k}: W_{j k}\right]} t_{k i}^{\left[W_{i j k}: W_{k i}\right]}=1 .
$$

Proof. By definition, $\left\{t_{i j}\right\} \in \Theta$ iff the associated algebra $A_{u^{+}}$is flat. Since this has the form of a twisted group algebra, this may be restated grouptheoretically as follows. Let $F$ be the free group on $r$ elements $s_{1}, \ldots, s_{r}$, and let $G:=\mathbb{C}^{*} \ltimes F$, where each generator of $F$ acts on $\mathbb{C}^{*}$ by $x \mapsto x^{-1}$. Finally, let $N$ be the normal subgroup of $G$ generated by the elements

$$
\begin{array}{rr}
s_{i}^{2}, & 1 \leq i \leq r \\
(-1)^{m_{i j}+1} t_{i j}\left(s_{i} s_{j}\right)^{-m_{i j},} & 1 \leq i<j \leq r .
\end{array}
$$

Then $t_{i j} \in \Theta$ iff $N$ is disjoint from $\mathbb{C}^{*}$, or equivalently iff there exists a (noncentral) extension of $W$ by $\mathbb{C}^{*}$ having preimages of the generators satisfying the above relations. The main theorem of $[\mathrm{Bu}]$ gives necessary and sufficient conditions for extensions of Coxeter groups by arbitrary $\mathbb{Z}$-modules; specializing Burichenko's condition to the present case gives the desired result.

Note that the group $Z$ acts on $\Theta$ via $t_{i j} \rightarrow t_{i j} z_{i j}^{m_{i j}}$. It is easy to see that the map $\psi$ descends to a map $\psi: \Theta / Z \rightarrow H^{2}\left(W_{+}, \mathbb{C}^{*}\right)$, which is injective. Let us show that this map is in fact a bijection. To do so, we will construct the inverse map $\eta$. Let $\psi$ be a Schur multiplier for $W_{+}$, and $\mathbb{C}_{\psi}\left[W_{+}\right]$be the corresponding twisted group algebra. Let $a_{i j}^{\mathrm{gr}}$ be the elements $a_{i j}$ in the group $W_{+}$as opposed to the algebra $\mathbb{C}_{\psi}\left[W_{+}\right]$. Let $0 \in I$ be an element. Define $a_{0 j}:=\left[a_{0 j}^{\mathrm{gr}}\right]$ for $j \neq 0, a_{j 0}:=a_{0 j}^{-1}$, and $a_{i j}:=a_{0 i}^{-1} a_{0 j}$ for $i \neq 0, j \neq 0$. Then $a_{i j}^{m_{i j}}=t_{i j}$, where $t_{i j}=t_{i j}(\psi)$. Thus we have attached to $\psi$ an element $t$ of $\Theta$ (and thus of $\Theta / Z$ ); we set $t=\eta(\psi)$. It is easy to see that $\eta$ is the desired inverse.

As a by-product, we see that $\Theta / Z$ is a group under multiplication, and $\psi, \eta$ are group isomorphisms.

In the sequel, a particular class in $H^{2}\left(W_{+}, \mathbb{C}^{*}\right)$ will be especially important. It is the Schur multiplier $\psi_{\text {Spin }}$ afforded by the pullback of a spinor representation of (the reductive part of) $S O(V)$ under the homomorphism $\rho: W_{+} \rightarrow S O(V)$.

Lemma 3.2. If $\psi=\psi_{\text {spin }}$ then $\eta(\psi)$ is the class of $t=1$, (i.e. $t_{i j}=1$ for all $i, j)$.

Proof. The representation $\rho: W \rightarrow O(V)$ lifts to a projective representation $\hat{\rho}: W \rightarrow \operatorname{Pin}(V)$. This representation is defined by the formula $\hat{\rho}\left(s_{i}\right)=e_{i}$. We have $e_{i}^{2}=1$, and $e_{i} e_{j}+e_{j} e_{i}=-2 \cos \left(\pi / m_{i j}\right)$. For $m_{i j}<\infty$, let $Y_{i j}$ be the 2-dimensional spin representation of the Clifford algebra generated by $e_{i}, e_{j}$. Then it follows from the last equation that the trace of $e_{i} e_{j}$ in this representation is $-2 \cos \left(\pi / m_{i j}\right)$. Thus the eigenvalues of $e_{i} e_{j}$ in $Y_{i j}$ are $-e^{ \pm \pi \sqrt{-1} / m_{i j}}$, and hence $\left(e_{i} e_{j}\right)^{m_{i j}}=(-1)^{m_{i j}+1}$, as desired. 


\section{ThE FlatNess LOCUS FOR FINITE TRIANGLES}

In [ER, it was shown that if all triangular (i.e., rank 3 parabolic) subgroups of $W$ are infinite, then $\mathbb{T}_{f}=\mathbb{T}$; that is, all parameters are flat. This suggests that in general, finite triangles will play a particularly important role.

It is a classical result that the triangle group with exponents $p, q, r$ is finite if and only if $1 / p+1 / q+1 / r>1$, and thus the only finite triangle groups are the infinite family

$$
\left\langle x, y, z \mid x^{2}=y^{2}=z^{n}=x y z=1\right\rangle, \quad 2 \leq n
$$

and the three sporadic examples

$$
\left\langle x, y, z \mid x^{2}=y^{3}=z^{n}=x y z=1\right\rangle, \quad 3 \leq n \leq 5 .
$$

We may thus proceed by a case-by-case analysis.

Since we are dealing with a finite algebra, the flatness condition at a point $u$ is equivalent to the requirement that $\operatorname{dim}\left(A_{u+}\right)=\left|W^{+}\right|$. If $A_{u+}$ is semisimple, this can be verified by exhibiting sufficiently many irreducible representations (with dimensions satisfying $\sum d^{2}=\left|W^{+}\right|$). This suffices in particular to determine any component of $\mathbb{T}_{f}$ for which the generic point is semisimple, or equivalently any component that contains any semisimple point. In particular, the group algebra itself is such a point, and thus gives rise to a large portion of the flatness locus. The representation theory of the group algebra is obviously relevant, and we must therefore distinguish in the infinite family between the cases $n$ even and odd.

Lemma 4.1. Suppose $n \geq 2$ is even, and consider the algebra $A_{u+}$ with $m_{12}=m_{23}=2, m_{13}=n$. If

$$
\begin{aligned}
t_{122} t_{232} & =t_{13 n}, \\
t_{121} t_{231} & =t_{13 n}, \\
t_{122} t_{231} & =t_{13(n / 2)}, \\
t_{121} t_{232} & =t_{13(n / 2)}, \\
t_{121} t_{122} t_{231} t_{232} & =t_{13 i} t_{13(n-i)}, \quad 1 \leq i<n / 2
\end{aligned}
$$

then $A_{u+}$ is flat. Similarly, if $n \geq 3$ is odd, $A_{u+}$ is flat if

$$
\begin{aligned}
t_{122} t_{232} & =t_{13 n} \\
t_{121} t_{231} & =t_{13 n} \\
t_{121} t_{122} t_{231} t_{232} & =t_{13 i} t_{13(n-i)}, \quad 1 \leq i<n / 2 .
\end{aligned}
$$


For the exceptional cases $m_{12}=2, m_{23}=3, m_{13}=n$, the algebra is flat if $n=3$ and

$$
\begin{aligned}
t_{122} t_{233} & =t_{133} \\
t_{122} t_{231} & =t_{131} \\
t_{122} t_{232} & =t_{132} \\
t_{121}^{2} t_{122} t_{231} t_{232} t_{233} & =t_{131} t_{132} t_{133} ;
\end{aligned}
$$

if $n=4$ and

$$
\begin{aligned}
t_{122} t_{233} & =t_{134} \\
t_{121} t_{233} & =t_{132} \\
t_{121} t_{122} t_{231} t_{232} & =t_{132} t_{134} \\
t_{121} t_{122}^{2} t_{231} t_{232} t_{233} & =t_{131} t_{132} t_{133} \\
t_{121}^{2} t_{122} t_{231} t_{232} t_{233} & =t_{131} t_{133} t_{134} ;
\end{aligned}
$$

or if $n=5$ and

$$
\begin{aligned}
t_{122} t_{233} & =t_{135} \\
t_{121} t_{122}^{2} t_{231} t_{232} t_{233} & =t_{131} t_{134} t_{135} \\
t_{121} t_{122}^{2} t_{231} t_{232} t_{233} & =t_{132} t_{133} t_{135} \\
t_{121}^{2} t_{122}^{2} t_{231} t_{232} t_{233}^{2} & =t_{131} t_{132} t_{133} t_{134} \\
t_{121}^{2} t_{122}^{3} t_{231}^{2} t_{232}^{2} t_{233} & =t_{131} t_{132} t_{133} t_{134} t_{135} .
\end{aligned}
$$

Proof. Note that in each case, the equations cut out a subgroup scheme of $\mathbb{T}$, and each of the (at most 2) components of the subgroup is smooth and has points in characteristic 0 . Since $\mathbb{T}_{f}$ is a closed subscheme of $\mathbb{T}$, we may thus work over $\mathbb{C}$.

It then suffices to show that the irreducible representations of $W_{+}$deform, for generic solutions of the above equations; more precisely, we claim that each equation gives the condition for such deformation. For instance, to deform the 5-dimensional representation of the $(2,3,5)$ group, we must have matrices $A$ and $B$ such that $A$ has eigenvalues $t_{121}$ and $t_{122}$ with multiplicities 2 and $3, B$ has eigenvalues $t_{231}, t_{232}, t_{233}$ with multiplicities 2,2 , and 1 , and $A B$ has eigenvalues $t_{13 i}$ with multiplicity 1 . This is an example of a rigid multiplicative Deligne-Simpson problem, and it follows from $[\underline{S}$ that such a solution exists (and is unique up to conjugacy) if and only if the determinants multiply up correctly.

Remark 4.1. The rigid Deligne-Simpson problems that arise above and in Lemma 4.2 are those with generically diagonalizable matrices with eigenvalue multiplicities from the list

$$
\begin{aligned}
& (1,1,1),(11,11,11),(21,111,111), \\
& (22,211,1111),(32,221,11111),(33,222,21111) .
\end{aligned}
$$


Now, in most of these cases, there is another way to structure the representations to obtain the correct dimensions; the point is that aside from the case $(2,2, n), n$ odd, the group has a nontrivial central extension (the spin extension discussed above), giving rise to a nontrivially twisted group algebra.

Lemma 4.2. Suppose $n \geq 2$ is even, and consider the algebra $A_{u+}$ with $m_{12}=m_{23}=2, m_{13}=n$. If

$$
t_{121} t_{122} t_{231} t_{232}=t_{13 i} t_{13(n+1-i)}, \quad 1 \leq i \leq n / 2,
$$

then $A_{u+}$ is flat. Similarly, the algebra is flat if $m_{12}=2, m_{23}=3, m_{13}=3$, and

$$
\begin{aligned}
& t_{121} t_{122} t_{231} t_{232}=t_{131} t_{132} \\
& t_{121} t_{122} t_{231} t_{233}=t_{131} t_{133} \\
& t_{121} t_{122} t_{232} t_{233}=t_{132} t_{133}
\end{aligned}
$$

if $m_{12}=2, m_{23}=3, m_{13}=4$, and

$$
\begin{aligned}
t_{121} t_{122} t_{231} t_{233} & =t_{131} t_{134} \\
t_{121} t_{122} t_{231} t_{233} & =t_{132} t_{133} \\
t_{121}^{2} t_{122}^{2} t_{231} t_{232}^{2} t_{233} & =t_{131} t_{132} t_{133} t_{134}
\end{aligned}
$$

or if $m_{12}=2, m_{23}=3, m_{13}=5$, and

$$
\begin{aligned}
t_{121} t_{122} t_{231} t_{232} & =t_{131} t_{135} \\
t_{121} t_{122} t_{231} t_{232} & =t_{132} t_{133} \\
t_{121}^{2} t_{122}^{2} t_{231} t_{232}^{2} t_{233} & =t_{131} t_{132} t_{134} t_{135} \\
t_{121}^{3} t_{122}^{3} t_{231}^{2} t_{232}^{2} t_{233}^{2} & =t_{131} t_{132} t_{133}^{2} t_{134} t_{135} .
\end{aligned}
$$

Proof. The proof is analogous to the proof of the previous lemma.

We can of course obtain other tori inside the flatness locus by permuting the $t_{i j k}$ for each $i j$; the claim, however, is that any flat choice of parameters lies inside one of these tori. We thus wish to show that $\mathbb{T}_{f}$ is the union of a quite large number of tori; the resulting equations would therefore be extremely complicated in general. However, if we mod out by the natural permutation action, we obtain a much simpler expression for the ideal. In other words, we need to consider the algebra $\tilde{A}_{+}$, or equivalently the algebra with relations

$$
\begin{array}{r}
a b c-1=a^{2}-\alpha_{1} a+\alpha_{2}=b^{2}-\beta_{1} b+\beta_{2}=0, \\
c^{n}-\gamma_{1} c^{n-1}+\gamma_{2} c^{n-2}-\cdots+(-1)^{n} \gamma_{n}=0,
\end{array}
$$

replacing the polynomial $b^{2}-\beta_{1} b+\beta_{2}$ with $b^{3}-\beta_{1} b^{2}+\beta_{2} b-\beta_{3}$ in the exceptional cases. 
Theorem 4.1. The flatness locus for the algebra $\tilde{A}_{+}$is defined by the following equations. For $(2,2, n), n \geq 2$ even:

$$
\begin{aligned}
\left(\alpha_{2} \beta_{2}\right)^{n} \gamma_{n}^{2} & =1 \\
\left(\alpha_{2} \beta_{2}\right)^{k} \gamma_{n} \gamma_{k} & =\gamma_{n-k} \\
\left(\alpha_{2} \beta_{2}\right)^{n / 2} \gamma_{n} \alpha_{1} & =\alpha_{1} \\
\left(\alpha_{2} \beta_{2}\right)^{n / 2} \gamma_{n} \beta_{1} & =\beta_{1} ;
\end{aligned}
$$

for $(2,2, n), n \geq 3$ odd:

$$
\begin{aligned}
\left(\alpha_{2} \beta_{2}\right)^{n} \gamma_{n}^{2} & =1 \\
\left(\alpha_{2} \beta_{2}\right)^{k} \gamma_{n} \gamma_{k} & =\gamma_{n-k} ; \\
\alpha_{2}^{(n-1) / 2} \beta_{2}^{(n+1) / 2} \gamma_{n} \alpha_{1} & =\beta_{1} ;
\end{aligned}
$$

for $(2,3,3)$ :

$$
\begin{aligned}
\alpha_{2}^{6} \beta_{3}^{4} \gamma_{3}^{4} & =1 \\
\alpha_{2}^{3} \beta_{3}^{2} \gamma_{3}^{2} \alpha_{1} & =\alpha_{1} \\
\alpha_{2}^{2} \beta_{3}^{2} \gamma_{3} \beta_{1} & =\gamma_{2} \\
\alpha_{2}^{2} \beta_{3} \gamma_{3}^{2} \gamma_{1} & =\beta_{2} ;
\end{aligned}
$$

for $(2,3,4)$ :

$$
\begin{aligned}
\alpha_{2}^{12} \beta_{3}^{8} \gamma_{4}^{6} & =1 \\
\alpha_{2}^{6} \beta_{3}^{4} \gamma_{4}^{3} \alpha_{1} & =\alpha_{1} \\
\alpha_{2}^{6} \beta_{3}^{4} \gamma_{4}^{3} \gamma_{2} & =\gamma_{2} \\
\alpha_{2}^{4} \beta_{3}^{3} \gamma_{4}^{2} \beta_{1} & =\beta_{2} \\
\alpha_{2}^{3} \beta_{3}^{2} \gamma_{4}^{2} \gamma_{1} & =\gamma_{3} ;
\end{aligned}
$$

and for $(2,3,5)$ :

$$
\begin{aligned}
\alpha_{2}^{30} \beta_{3}^{20} \gamma_{5}^{12} & =1 \\
\alpha_{2}^{15} \beta_{3}^{10} \gamma_{5}^{6} \alpha_{1} & =\alpha_{1} \\
\alpha_{2}^{10} \beta_{3}^{7} \gamma_{5}^{4} \beta_{1} & =\beta_{2} \\
\alpha_{2}^{6} \beta_{3}^{4} \gamma_{5}^{3} \gamma_{1} & =\gamma_{4} \\
\alpha_{2}^{12} \beta_{3}^{8} \gamma_{5}^{5} \gamma_{2} & =\gamma_{3} .
\end{aligned}
$$

Proof. To show that the equations are sufficient for flatness, we note that over $\mathbb{C}$ the above equations imply simple multiplicative relations between the roots of the minimal polynomials (e.g., the fact that if the polynomial $p$ satisfies $p(x)=(-1)^{n} x^{n} p(1 / x)$, then its roots multiply pairwise to 1$)$, which in turn imply flatness, using the appropriate lemma. In particular, since flatness is a closed condition, this proves sufficiency over $\mathbb{Z}$. 
Now let us prove the necessity of the equations. In the exceptional cases, we can simply compute (using MAGMA) a noncommutative Gröbner basis for $\tilde{A}_{+}$over $R$, ignoring any reduced $S$-polynomials which vanish in the group case; it turns out that the remaining $S$-polynomials have invertible leading coefficients once reduced. We thus find that every element of the Gröbner basis in the group case can be lifted to a monic relation in $\tilde{A}_{+}$, and thus every remaining $S$-polynomial must reduce to 0 modulo those relations. The coefficients of such reductions thus give equations for the flatness locus.

For the infinite family, essentially the same idea holds; since in that case, the computations must be done by hand, it is more convenient to work with $\tilde{A}$, and stop as soon as the above equations are obtained.

Corollary 4.1. In each case $\mathbb{T}_{f}$ is the union of the relevant schemes from Lemmas 4.1 and 4.2 .

Remark 4.2. The key observation to make about the above equations is that they are linear in the middle coefficients, and thus the fibers of the map from the flatness locus to the locus of twisted group algebras are connected. Moreover, in characteristic not 2, the corresponding vector spaces can be given a basis depending only on the component; that is, the flatness locus is a vector bundle over the locus of twisted group algebras. Note that the fiber over a nonreduced point will still be an affine space, but such a point fails to have even a formal neighborhood over which the scheme remains an affine space.

Now consider the case of Coxeter groups of rank higher than 3. For any triple of distinct indices $\Delta=\{i, j, k\} \subset I$, let $M_{\Delta}$ be the corresponding 3 by 3 submatrix of $M$, and $W_{\Delta}=W\left(M_{\Delta}\right)$. Let $\mathbb{T}^{\Delta}$ be the corresponding torus of parameters; we have a natural projection $p=p_{\Delta}: \mathbb{T} \rightarrow \mathbb{T}^{\Delta}$.

Let $A_{+}^{\Delta}:=A_{+}\left(M_{\Delta}\right)$. Let $J_{\Delta} \subset R$ be the intersection of all ideals $I$ in $R$ such that $T_{w(x)}, x \in W_{\Delta+}$, are a basis of $A_{+}^{\Delta} / I A_{+}^{\Delta}$ over $R / I$, and let $\mathbb{T}_{f}^{\Delta}=\operatorname{Spec} R / J_{\Delta}($ a closed subscheme of $\mathbb{T})$. If $k$ is a field then $\mathbb{T}_{f}^{\Delta}(k) \subset \mathbb{T}(k)$ be the locus of points $t \in \mathbb{T}(k)$ such that $T_{w(x)}, x \in W_{\Delta+}$ are a basis of $A_{p(t)+}^{\Delta}$. Also, let $\tilde{\mathbb{T}}_{f}^{\Delta}=\mathbb{T}_{f}^{\Delta} / \prod_{i<j} S_{m_{i j}}$.

Since the equations on the middle coefficients are all of the form $p(x)=$ $a q(b x)$ or $p(x)=a x^{n} q(b / x)$ where $p, q$ are minimal polynomials of the generators and $a, b$ are monomials in the constant coefficients, it follows that similar comments to Remark 4.2 apply to the scheme $\cap_{\Delta \subset I} \tilde{\mathbb{T}}_{f}^{\Delta}$. Indeed, the equations on the middle coefficients are still linear, and the only way the dimension can depend on the constant coefficients is via equations of the form $p(x)=p(a x)$ with $a^{n}=1$ or $p(x)=p(0)^{-1} x^{n} p(a / x)$ with $a^{n}=p(0)^{2}$. Thus in sufficiently large characteristic (e.g., $p>m_{i j}$ whenever $m_{i j}<\infty$ ), the intersection $\cap_{\Delta \subset I} \tilde{\mathbb{T}}_{f}^{\Delta}$ is still a vector bundle.

More generally, it follows from the above discussion that over a sufficiently large cyclotomic ring (so the twisted group locus breaks into smooth 
components), the scheme $\cap_{\Delta \subset I} \tilde{\mathbb{T}}_{f}^{\Delta}$ can be expressed as a union of smooth components, one for each component of the twisted group locus. Since the polynomials associated to a point on the twisted group locus have distinct roots in a suitable cyclotomic ring, the scheme $\cap_{\Delta \subset I} \mathbb{T}_{f}^{\Delta}$ is also a union of smooth components, a total of $\left|\prod_{i<j} S_{m_{i j}}\right|$ for each component of the twisted group locus.

\section{The Structure of the Flatness locus AROUnd A 2-COCyCle}

5.1. The formal PBW theorem. Let $\mathbb{T}_{f}^{*}=\cap_{\Delta} \mathbb{T}_{f}^{\Delta}$. It is clear that $\mathbb{T}_{f} \subset$ $\mathbb{T}_{f}^{*}$. Moreover, by Proposition 3.1 and the explicit computations of $\mathbb{T}_{f}^{\Delta}$ in the previous section, it follows that every component of $\mathbb{T}_{f}^{*}$ is integral and contains a complex 2-cocycle $\psi \in \Theta$. Theorem 1.1] will thus follow if we manage to show that every point of $\mathbb{T}_{f}^{*}$ in some neighborhood of $\psi$ is actually in $\mathbb{T}_{f}$.

The main result of this section, which accomplishes this, is Theorem 5.1 below. Note that it is enough for us to work over $\mathbb{C}$; we will do so, and for simplicity will use the same notation for the complex counterparts of the $\mathbb{Z}$-objects considered before.

Theorem 5.1. Let $\psi \in \Theta$. Then there exists a formal neighborhood $U$ of $\psi$ in $\mathbb{T}$ such that $\mathbb{T}_{f} \cap U=\mathbb{T}_{f}^{*} \cap U$.

The rest of the section is devoted to the proof of Theorem 5.1

Let $\hat{R}, \hat{A}_{+}$be the completions of $R, A_{+}$near the point $\psi$. Let $J \subset \hat{R}$ be the completion of the ideal of $\mathbb{T}_{f}^{*}$. Our job is to show that the elements $T_{w(x)}, x \in W_{+}$, form a (topological) basis of the algebra $\hat{A}_{+} / J \hat{A}_{+}$over $\hat{R} / J$. This will be done using the theory of sheaves on posets (which is very closely related to the constructible sheaves used in [ER], but is more elementary). We let $t_{\psi}=t$ be the collection of numbers $t_{i j}$ attached to $\psi$ as above.

5.2. The cell complex $\Sigma$ attached to a Coxeter group. Let us recall (see e.g. Da that to any Coxeter group $W=W(M)$ one can attach a regular cell complex $\Sigma=\Sigma(M)$ as follows. The cells of dimension $n$ in $\Sigma$ are labelled by cosets in $W$ of a finite parabolic subgroup $W_{Q}$ corresponding to an $n$-element subset $Q \subset I$. The boundary of the cell $\sigma \in W / W_{Q}$ consists of the cells $\sigma^{\prime} \in W / W_{Q^{\prime}}, Q^{\prime} \subset Q$, such that $\sigma^{\prime} \subset \sigma$. Also, for every $Q$ we have a canonical cell $\sigma_{Q} \in W / W_{Q}$ containing the identity element of $W$.

Theorem 5.2. (see e.g. [Da]) The cell complex $\Sigma$ is regular and contractible. The group $W$ acts on $\Sigma$ properly discontinuously.

Let $\Sigma_{n}$ denote the $n$-skeleton of $\Sigma$. It follows from Theorem 5.2 that $H^{i}\left(\Sigma_{n}, \mathbb{C}\right)=0$ for $i<n$.

5.3. Equivariant sheaves on posets. Given a poset $P$, a sheaf on $P$ is a functor from $P$ to Vect; that is, an assignment of a (complex) vector space $S(x)$ for $x \in P$, together with maps $f_{x y}: S(y) \rightarrow S(x)$ for $y \subset x$, satisfying 
$f_{x y} \circ f_{y z}=f_{x z}$. If the maps $f_{x y}$ are isomorphisms, we say that $S$ is a local system. If $V$ is a vector space, then there is a local system (the constant sheaf) with $S(x) \equiv V$ and $f_{x y} \equiv 1$; we will denote this sheaf by $V$.

Proposition 5.1. (see e.g. $\mathrm{Yu}$ ) There is a canonical isomorphism

$$
\operatorname{Ext}_{P}^{*}(\mathbb{C}, \mathbb{C}) \cong H^{*}(\Delta(P)),
$$

where $\Delta(P)$ is the order complex of $P$, and Ext $t_{P}^{*}$ is taken in the category of sheaves on $P$.

In particular, if $P$ is the poset of closed cells of a regular $\mathrm{CW}$ complex $\Sigma$, then $\Delta(P)$ is homeomorphic to the barycentric subdivision of $\Sigma$, and we have $\operatorname{Ext}_{P}^{*}(\mathbb{C}, \mathbb{C}) \cong H^{*}(\Sigma)$.

Now, suppose the group $G$ acts on $P$ (preserving order), and let $\psi$ be a 2-cocycle of $G$. We can then define a $\psi$-equivariant sheaf to be a sheaf on $P$ together with maps

$$
\rho(g): S(x) \rightarrow S(g x)
$$

compatible with the sheaf maps and satisfying

$$
\rho(g) \rho(h)=\psi(g, h) \rho(g h) .
$$

Note that for each $x \in P, S(x)$ is a $\mathbb{C}_{\psi}\left[G_{x}\right]$-module, and the maps $f_{x y}$ are $\mathbb{C}_{\psi}\left[G_{x} \cap G_{y}\right]$-linear.

The forgetful functor $\pi^{*}$ from $\psi$-equivariant sheaves to ordinary sheaves has a natural adjoint, which associates to the sheaf $V$ the sheaf

$$
\left(\pi_{!} V\right)_{x}=\bigoplus_{g \in G} V_{g^{-1} x}
$$

such that

$$
\rho(g):\left(\pi_{!} V\right)_{x} \rightarrow\left(\pi_{!} V\right)_{g x}
$$

acts on $V_{h^{-1} x} \cong V_{(g h)^{-1} g x}$ as multiplication by $\psi(g, h)$.

5.4. Equivariant sheaves on the 3-skeleton of $\Sigma$. In our case, we are primarily interested in $\psi$-equivariant sheaves on $\Sigma_{3}$, where $\psi$ is a 2-cocycle of $W_{+}$. To describe them explicitly, we first need to understand the structure of $Y_{3}:=\Sigma_{3} / W_{+}$. It consists of the following cells

0-cells: $N, S$ (north and south pole).

1-cells: $e_{i}, i \in I$, connnecting $N$ and $S$.

2-cells: $D_{i j}, i<j$, a disk whose boundary is the union of $e_{i}$ and $e_{j}$, if $m_{i j}<\infty$ (for convenience we fix an identification $I=\{1, \ldots, r\}$ ). The center of $D_{i j}$ has isotropy group $W_{i j+}=\mathbb{Z}_{m_{i j}}$.

3-cells: $P_{i j k}, i<j<k$, a 3-ball whose boundary is the sphere made up by $D_{i j}, D_{j k}, D_{i k}$, if $W_{i j k}$ is a finite group. The center of $P_{i j k}$ has isotropy group $W_{i j k+}$, and the points on the segments connecting the center of $P_{i j k}$ with the centers of $D_{i j}, D_{j k}, D_{i k}$ have isotropy groups $W_{i j+}=\mathbb{Z}_{m_{i j}}, W_{j k+}=\mathbb{Z}_{m_{j k}}$, $W_{i k+}=\mathbb{Z}_{m_{i k}}$.

Remark. More precisely, the 3-cells are of the form $B^{3} / W_{i j k+}$, where $B^{3}$ is the 3 -ball. But the quotient $B^{3} / G$ for $G \subset S O(3, \mathbb{R})$ is always 
isomorphic to $B^{3}$ as a topological space, since $G \subset P S L_{2}(\mathbb{C})$ and hence $S^{2} / G=\mathbb{C} P^{2} / G=\mathbb{C} P^{1}=S^{2}$.

Thus, $\psi$-equivariant sheaves on $\Sigma_{3}$ can be specified by the following data:

1. Spaces $V_{N}, V_{S}$

2. A space $V_{i}$ for all $i \in I$, and maps $f_{N i}: V_{N} \rightarrow V_{i}, f_{S i}: V_{S} \rightarrow V_{i}$.

3. A $\mathbb{C}_{\psi}\left[W_{i j+}\right]$-module $V_{i j}=V_{j i}$ for $i \neq j \in I$, and maps $h_{i j}: V_{i} \rightarrow V_{i j}$.

4. A $\mathbb{C}_{\psi}\left[W_{i j k+}\right]$-module $V_{i j k}=V_{i k j}=\cdots$ for $i, j, k \in I$ distinct, and $\mathbb{C}_{\psi}\left[W_{i j+}\right]$-linear maps

$$
f_{i j ; k}: V_{i j} \rightarrow \operatorname{Res}_{W_{i j+}}^{W_{i j k+}} V_{i j k}
$$

subject to the obvious compatibility relations on the maps, and the omission of spaces $V_{i j}$ and $V_{i j k}$ corresponding to infinite parabolic subgroups.

Let $\mathcal{C}$ denote the category of sheaves on the cell poset of $\Sigma_{3}$, and let $\mathcal{C}_{\psi}$ denote the corresponding category of $\psi$-equivariant sheaves. Finally, let $\mathbf{M}$ be the $\psi$-equivariant local system $\pi_{!}(\mathbb{C})$.

Lemma 5.1. One has $\operatorname{Ext}_{\mathcal{C}_{\psi}}^{j}(\mathbf{M}, \mathbf{M})=0$ for $j=1,2$.

Proof.

$$
\begin{gathered}
\operatorname{Ext}_{\mathcal{C}_{\psi}}^{j}(\mathbf{M}, \mathbf{M})=\operatorname{Ext}_{\mathcal{C}_{\psi}}^{j}\left(\pi ! \mathbb{C}, \pi_{!} \mathbb{C}\right)= \\
\operatorname{Ext}_{\mathcal{C}}^{j}\left(\mathbb{C}, \pi^{*} \pi_{!} \mathbb{C}\right)=\mathbb{C}_{\pi}\left[W_{+}\right] \otimes \operatorname{Ext}_{\mathcal{C}}^{j}(\mathbb{C}, \mathbb{C})= \\
\mathbb{C}_{\pi}\left[W_{+}\right] \otimes H^{j}\left(\Sigma_{3} ; \mathbb{C}\right)=0 .
\end{gathered}
$$

5.5. The algebra $B$. The category $\mathcal{C}_{\psi}$ is abelian, and is thus equivalent to the category of modules over some algebra, which can in fact be read off from the above description of the data determining a $\psi$-equivariant sheaf. In particular, the representations of $B$ correspond to sums

$$
V_{N} \oplus V_{S} \oplus \bigoplus_{i} V_{i} \oplus \bigoplus_{i, j} V_{i j} \oplus \bigoplus_{i, j, k} V_{i j k}
$$

as above.

Now, let $\tau=\left(\tau_{i j k}\right), k \in \mathbb{Z}_{m_{i j}}$, be a collection of formal parameters. If we replace each algebra $\mathbb{C}_{\psi}\left[W_{i j+}\right], \mathbb{C}_{\psi}\left[W_{i j k+}\right]$ by the corresponding deformation $A_{i j+}, A_{i j k+}$, we obtain a deformed algebra $B(\tau)$, over $\hat{R}:=\mathbb{C}[[\tau]]$. Let $J$ be the ideal of the formal neighborhood of $\mathbb{T}_{f}^{*}$ in $\hat{R}$, and let $B_{J}(\tau)=$ $B(\tau) / J B(\tau)$ be the corresponding algebra over $\hat{R}_{J}:=\hat{R} / J$.

Proposition 5.2. For any Coxeter matrix $M$, the algebra $B_{J}(\tau)$ is a flat deformation of $B$ over the formal neighborhood of $t=\eta(\psi)$ in $\mathbb{T}_{f}^{*}$.

Proof. For any set $i, j$ of distinct indices, let $p_{i j}$ be the idempotent of $B$ which acts by 1 on $V_{N}, V_{S}, V_{i}, V_{j}$, on $V_{i j}$ if $m_{i j}<\infty$, and by 0 on all the other spaces. Also, for any triangle $i, j, k$ such that $W_{i j k}$ is finite, let $p_{i j k}$ be the idempotent of $B$ which acts by 1 on $V_{N}, V_{S}, V_{i}, V_{j}, V_{k}, V_{i j}, V_{j k}, V_{i k}, V_{i j k}$, and by 0 on all the other spaces. 
Let $N$ be the direct sum of right $B$-modules $p_{i j k} B$ and $p_{i j} B$. Then $N$ is clearly a faithful right $B$-module, so it suffices to show that the modules $p_{i j k} B$ and $p_{i j} B$ can be deformed to right $B_{J}(\tau)$-modules. We focus on $p_{i j k} B$; the case of $p_{i j} B$ is analogous (and was in any case treated in [ER, Proposition 3.10).

Clearly, $B$ preserves the kernel of $p_{i j k}$, so $p_{i j k} B=p_{i j k} B p_{i j k}$, and thus $B_{i j k}:=p_{i j k} B$ is a unital algebra with unit $p_{i j k}$. Moreover, both $p_{i j k} B$ and $p_{i j k} B(\tau)$ are simply the analogues of $B$ for the rank 3 subsystem $\{i, j, k\}$. We may thus assume without loss of generality that $M$ has rank 3 . Then, to show that $B(\tau)$ is flat, it suffices to find a faithful representation which deforms flatly. The local system $\pi_{!}(\mathbb{C})$ affords such a representation, since by assumption $\mathbb{C}_{\psi}\left[W_{+}\right]$deforms flatly to $\hat{A}_{+}(M) / J \hat{A}_{+}(M)$.

5.6. End of proof of Theorem [5.1. Now we can finish the proof of Theorem 5.1 We can regard $\mathbf{M}$ as a $B$-module (in which all the arrows are isomorphisms). By Lemma 5.1] $\operatorname{Ext}_{B}^{1}(\mathbf{M}, \mathbf{M})=\operatorname{Ext}_{B}^{2}(\mathbf{M}, \mathbf{M})=0$. By standard deformation theory, this implies that $\mathbf{M}$ can be uniquely deformed to a module $\mathbf{M}(\tau)$ over $B_{J}(\tau)$. This module still has the property that all maps are isomorphisms. Thus the $\hat{A}_{i j k+} / J \hat{A}_{i j k+}$-module structure on the subspace $\mathbf{M}(\tau)_{i j k}$ transports to such a structure on the subspace $\mathbf{M}(\tau)_{N}$ (the fiber at the north pole), and all of the different module structures will be compatible. In other words, $\mathbf{M}(\tau)_{N}$ is a representation of $\hat{A}_{+} / J \hat{A}_{+}$that flatly deforms the regular representation of $\mathbb{C}_{\psi}\left[W_{+}\right]$. The existence of such a deformation implies the flatness of $\hat{A}_{+} / J \hat{A}_{+}$. Theorem 5.1 is proved.

\section{A generalization of the Iwahori-Hecke Algebra}

Although Theorem 1.1] is stated in terms of deformations of even subgroups of Coxeter groups, it also gives rise to interesting deformations of Coxeter groups themselves. Indeed, if $u \in \mathbb{T}_{f}$ is invariant under the $\mathbb{Z}_{2}$

action (modulo the permutation action), then $\tilde{A}_{\pi(u)}$ is an algebra deforming $\mathbb{Z}[W]$. The corresponding flatness locus is obtained from $\mathbb{T}_{f}$ by adding the additional "edge" conditions

$$
\left(e_{i j}^{\left(m_{i j}\right)}\right)^{2}=1, e_{i j}^{(k)}=e_{i j}^{\left(m_{i j}\right)} e_{i j}^{\left(m_{i j}-k\right)} .
$$

Of particular interest is the case $e_{i j}^{\left(m_{i j}\right)}=(-1)^{m_{i j}-1}, e_{i j}^{\left(m_{i j} / 2\right)}=0$ (the latter being redundant when 2 is invertible). It turns out that in this case, the flatness conditions are greatly simplified: only the $(2,3,3)$ case gives an additional condition, namely that if $m_{i j}=m_{j k}=3, m_{i k}=2$, then

$$
e_{i j}^{(1)}=e_{j k}^{(1)} .
$$

This gives rise to the following generalization of the Iwahori-Hecke algebra. If $x, y$ are elements of an associative algebra, let $B_{k}(x, y)$ denote the corresponding braid relation; that is,

$$
B_{2 k}(x, y)=(x y)^{k}-(y x)^{k}, \quad B_{2 k+1}(x, y)=(x y)^{k} x-(y x)^{k} y .
$$


Theorem 6.1. Introduce $u_{i}, v_{i}, 1 \leq i \leq r$, and for $i<j$ such that $m_{i j}<$ $\infty$, introduce parameters $f_{i j}^{(l)}, 1 \leq l<m_{i j} / 2$, such that the following two conditions hold:

(1) If $m_{i j}<\infty$ is odd, then $u_{i}=u_{j}, v_{i}=v_{j}$.

(2) If $m_{i j}=m_{j k}=3, m_{i k}=2$, then $f_{i j}^{(1)}=f_{j k}^{(1)}$.

Let $A^{\prime}$ be the $\mathbb{Z}\left[u_{i}, v_{i}, f_{i j}^{(l)}\right]$-algebra with presentation

$$
\begin{aligned}
& \left\langle T_{1}, \ldots, T_{r}\right| T_{i}^{2}-u_{i} T_{i}+v_{i}, \\
& \left.\quad B_{m_{i j}}\left(T_{i}, T_{j}\right)+f_{i j}^{(1)} B_{m_{i j}-2}\left(T_{i}, T_{j}\right)+f_{i j}^{(2)} B_{m_{i j}-4}\left(T_{i}, T_{j}\right)+\cdots\right\rangle,
\end{aligned}
$$

Then $A^{\prime}$ is a free $\mathbb{Z}\left[u_{i}, v_{i}, f_{i j}^{(l)}\right]$-module, with basis of the form $T_{w}, w \in W$. In particular, $A^{\prime}$ is a flat deformation of $\mathbb{Z}[W]$.

Proof. If we choose a reduced word for each element $w \in W$, the corresponding products $T_{w}$ certainly span $A^{\prime}$, and it suffices to show that they are linearly independent. To show this, we may adjoin $1 / 2$ and $1 / \sqrt{u_{i}^{2}-4 v_{i}}$ for all $i$ to the coefficient ring. At this point, if we consider new generators $U_{i}=\left(2 T_{i}-u_{i}\right) / \sqrt{u_{i}^{2}-4 v_{i}}$ we need simply show that the corresponding $U_{w}$ are linearly independent (since the two sets are related by a triangular change of basis with unit diagonal). In terms of the $U_{i}$, the deformed braid relations have the same form (given condition (1) above), and thus it suffices to prove linear independence when $u_{i}=0, v_{i}=1$.

At this point, if we multiply the deformed braid relation by $\left(T_{j} T_{i}\right)^{m_{i j} / 2}$ or $T_{j}\left(T_{i} T_{j}\right)^{\left(m_{i j}-1\right) / 2}$ as appropriate, we obtain a polynomial in $T_{i} T_{j}$. But the resulting algebra is then a specialization $\tilde{A}_{\pi(u)}$ satisfying the invariance and flatness conditions.

In particular, the case $f_{i j}^{(l)} \equiv 0$ is the ordinary Iwahori-Hecke algebra associated to $W$.

Remark 6.1. Other 2-cocycles of $W$ will of course have other deformations associated to them; the difficulty, however, is that the translation by $u_{i} / 2$ no longer preserves the form of the deformed braid relation in those cases.

Another way of obtaining flat deformations of $\mathbb{Z}[W]$ is via the observation that every Coxeter group is also the even subgroup of a slightly larger Coxeter group: $W=\left(W \times \mathbb{Z}_{2}\right)_{+}$. The resulting algebras include all algebras of the form $\tilde{A}_{\pi(u)}$, as well as deformations in which the quadratic relations are more general. It is unclear, however, whether these deformations are truly more general; it is possible that the extra freedom can be removed as in the above Hecke algebra case.

\section{Additive Versions}

In this section, for simplicity, we work over $\mathbb{C}$ rather than over $\mathbb{Z}$. 
7.1. Graded algebras. Recall ER that the polynomial relation for the algebra $A$ can be written as

$$
s_{i} s_{j} \ldots=(-1)^{m_{i j}+1} t_{i j} s_{j} s_{i} \ldots+\text { S.L.T. }
$$

$\left(m_{i j}\right.$ factors on both sides), where $t_{i j}:=\prod_{k} t_{i j k}$, and S.L.T. stands for "smaller length terms". Thus it is natural to define the graded version $\bar{A}$ of the algebra $A$, generated over $\bar{R}:=\mathbb{C}\left[t_{i j}\right]\left(t_{j i}=t_{i j}^{-1}\right)$ by $s_{i}$ with defining relations

$$
s_{i}^{2}=0, s_{i} s_{j} \ldots=(-1)^{m_{i j}+1} t_{i j} s_{j} s_{i} \ldots, \quad s_{i} t_{j p}=t_{j p}^{-1} s_{i} .
$$

Let $\bar{A}_{t}$ be the fiber of $\bar{A}$ over $t \in \overline{\mathbb{T}}:=\operatorname{Spec} \bar{R}$ (not always an algebra).

It is easy to see that $T_{w(x)}, x \in W$, is still a spanning set of $\bar{A}$ over $\bar{R}$ and of $\bar{A}_{t}$ over $\mathbb{C}$, and that if $t \in \Theta$ then $T_{w(x)}$ are a basis of $\bar{A}_{t}$. Also, if $T_{w(x)}$ are a basis, the Hilbert series of $\bar{A}_{t}$ is equal to $h(z)=h_{M}(z)$.

Let us now consider the algebra $\bar{A}_{1}$, corresponding to the special case $t=1$. Note that by Lemma 3.2. $1 \in \Theta$, so in this case $T_{w(x)}$ are a basis.

Lemma 7.1. (i) The algebra $A_{1}$ is generated by $s_{i}$ with defining relations $s_{i}^{2}=1,\left(s_{i}-s_{j}\right)^{m_{i j}}=0$.

(ii) The algebra $\bar{A}_{1}$ is generated by $s_{i}$ with defining relations $s_{i}^{2}=0$, $\left(s_{i}-s_{j}\right)^{m_{i j}}=0$.

Proof. (i) We will show that the relation $\left(s_{i} s_{j}-1\right)^{m_{i j}}=0$ is equivalent to $\left(s_{i}-s_{j}\right)^{m_{i j}}=0$ in the presence of the relations $s_{i}^{2}=s_{j}^{2}=1$. To do so, we write

$\left(s_{i}-s_{j}\right)^{m_{i j}}=\left(s_{i} s_{j}-1\right) s_{j} * s_{i}\left(1-s_{i} s_{j}\right) *\left(s_{i} s_{j}-1\right) s_{j} \ldots= \pm\left(s_{i} s_{j}-1\right)^{m_{i j}} s_{j} s_{i} s_{j} \ldots$, since $s_{j} s_{i}$ is the inverse of $s_{i} s_{j}$ and hence commutes with it. This proves (i).

(ii) The result follows by opening brackets in the relation $\left(s_{i}-s_{j}\right)^{m_{i j}}=0$, and writing down the resulting $2^{m_{i j}}$ terms.

7.2. Additive versions. Let us define the "additive" versions of the algebras $A, A_{+}$. Namely, let $\tau_{i j k}$ be parameters, such that $\tau_{i j k}=-\tau_{j i,-k}$, $k \in \mathbb{Z}_{m_{i j}}\left(m_{i j}<\infty\right)$.

Define the algebra $\mathcal{A}_{+}$generated over $\mathcal{R}:=\mathbb{C}[\tau]$ by generators $\alpha_{i j}, i \neq j$, with defining relations

$$
\alpha_{i j}+\alpha_{j i}=0, \alpha_{i j}+\alpha_{j k}+\alpha_{k i}=0, \prod_{k=1}^{m_{i j}}\left(\alpha_{i j}-\tau_{i j k}\right)=0\left(m_{i j}<\infty\right) .
$$

Further, define $\mathcal{A}$ to be the semidirect product $\mathbb{C Z}_{2} \ltimes \mathcal{A}_{+}$, where the nontrivial element $\sigma$ of $\mathbb{Z}_{2}$ acts as follows: $\sigma\left(\alpha_{i j}\right)=-\alpha_{i j}, \sigma\left(\tau_{i j k}\right)=\tau_{j i k}$. We'll denote by $\mathcal{A}_{0}, \mathcal{A}_{0+}$ the zero-fibers of these algebras. Thus, $\mathcal{A}_{0+}$ is generated by $\alpha_{i j}$ with defining relations

$$
\alpha_{i j}+\alpha_{j i}=0, \alpha_{i j}+\alpha_{j k}+\alpha_{k i}=0, \alpha_{i j}^{m_{i j}}=0\left(m_{i j}<\infty\right) .
$$

This algebra is naturally graded by $\mathbb{Z}_{+}\left(\operatorname{deg}\left(\alpha_{i j}\right)=1\right)$. 
Proposition 7.2. Let $0 \in I$. For any element $x \in W$ such that $l\left(s_{0} x\right)>$ $l(x)$, fix a reduced decomposition $x=s_{i_{1}} \ldots s_{i_{n}}$. Set $b_{x}:=\alpha_{0 i_{1}} \alpha_{i_{1} i_{2}} \ldots \alpha_{i_{n-1} i_{n}}$ (we agree that $b_{1}=1$ ). Then the collection of all the elements $b_{x}$ is a spanning set of $\mathcal{A}_{0+}$. In particular, the Hilbert series of $\mathcal{A}_{0+}$ is dominated (coefficientwise) by $h(z) /(1+z)$.

Proof. Since $\alpha_{i j}+\alpha_{j i}=0, \alpha_{i j}+\alpha_{j k}+\alpha_{k i}=0$, we see that any element of $\mathcal{A}_{0+}$ is a linear combination of elements of the form $\alpha_{0 i_{1}} \alpha_{i_{1} i_{2}} \ldots \alpha_{i_{n-1} i_{n}}$ for some $i_{1}, \ldots, i_{n}$.

Now let us apply the following "braid" relations of degree $m_{i j}$, which are clearly satisfied in $\mathcal{A}_{0+}$ :

if $m_{i j}$ is odd, and

$$
\begin{aligned}
\alpha_{i j} \alpha_{j i} \ldots \alpha_{j i} \alpha_{i p} & =\alpha_{j i} \alpha_{i j} \ldots \alpha_{i j} \alpha_{j p}, \\
\alpha_{p i} \alpha_{i j} \alpha_{j i} \ldots \alpha_{j i} & =\alpha_{p j} \alpha_{j i} \alpha_{i j} \ldots \alpha_{i j}
\end{aligned}
$$

$$
\begin{aligned}
\alpha_{i j} \alpha_{j i} \ldots \alpha_{i j} \alpha_{j p} & =-\alpha_{j i} \alpha_{i j} \ldots \alpha_{j i} \alpha_{i p}, \\
\alpha_{p i} \alpha_{i j} \alpha_{j i} \ldots \alpha_{i j} & =-\alpha_{p j} \alpha_{j i} \alpha_{i j} \ldots \alpha_{j i}
\end{aligned}
$$

if $m_{i j}$ is even. Using these braid relations, we can reduce the monomial $\alpha_{0 i_{1}} \alpha_{i_{1} i_{2}} \ldots \alpha_{i_{n-1} i_{n}}$ to zero if the word $s_{0} \ldots . s_{n}$ is not reduced, and to the monomial $b_{x}$ for some $x$ if this word is reduced. The proposition is proved.

Proposition 7.3. (i) There exists a (unique) homomorphism $\phi_{0}: \mathcal{A}_{0+} \rightarrow$ $\bar{A}_{1}$ defined by the formula $\phi_{0}\left(a_{i j}\right)=s_{i}-s_{j}$.

(ii) There exists a (unique) homomorphism $\phi: \mathcal{A}_{0+} \rightarrow A_{1}$ defined by the formula $\phi\left(a_{i j}\right)=s_{i}-s_{j}$.

Proof. This follows immediately from Lemma 7.1

Lemma 7.4. Let $B=I m \phi_{0}$, i.e. the subalgebra of $\bar{A}_{1}$ generated by $s_{i}-s_{j}$. Then for any $0 \in I, \bar{A}_{1}=B+s_{0} B$.

Proof. We first show that any element of $\bar{A}_{1}$ can be written as a linear combination $x+s_{0} y, x, y \in B$. It suffices to consider elements $z=s_{i_{1}} \ldots s_{i_{n}}$, and use induction in $n$. If $n=0,1$, the statement is clear $\left(s_{i}=s_{0}+\left(s_{i}-s_{0}\right)\right)$. If $n>1$, we have

$$
z=s_{i_{1}} \ldots s_{i_{n-1}} s_{i_{n}}=s_{i_{1}} s_{i_{2}} \ldots s_{i_{n-1}}\left(s_{i_{n}}-s_{i_{n-1}}\right) .
$$

Since $s_{1} \ldots s_{n-1} \in B+s_{0} B$ by the induction assumption, we get $s_{1} \ldots s_{n} \in$ $B+s_{0} B$.

Thus, $\bar{A}_{1}=B+s_{0} B$.

The main result of this section is the following theorem.

Theorem 7.5. (i) The map $\phi_{0}: \mathcal{A}_{0+} \rightarrow B$ is an isomorphism.

(ii) The Hilbert series of $\mathcal{A}_{0+}$ and $B$ is $h(z) /(1+z)$.

(iii) $\bar{A}_{1}=B \oplus s_{0} B$.

(iv) The spanning set of $\mathcal{A}_{0+}$ from Proposition 7.2 is in fact a basis. 
Proof. By Lemma 7.4, the Hilbert series of $B$ is at least $h(z) /(1+z)$, therefore so is the Hilbert series of $\mathcal{A}_{0+}$. On the other hand, by Proposition 7.2. the opposite inequality holds. This implies that the Hilbert series in question is exactly $h(z) /(1+z)$. (i)-(iv) immediately follow.

We conjecture that (ii) remains true for the fibers $\mathcal{A}_{\tau+}$ over the locus of $\tau$ such that (ii) holds for finite triangles; equivalently for $\tau$ in the tangent cone to $\mathbb{T}_{f}$ at 1 . This conjecture is true in the rank 3 case by using the RiemannHilbert correspondence between solutions of the additive and multiplicative Deligne-Simpson problems.

\section{REFERENCES}

[B] N. Bourbaki, Chapitres IV, V, VI of Groupes et algebres de Lie, Hermann, Paris, 1968.

[Bu] Burichenko, Vladimir P., On extensions of Coxeter groups, Comm. Algebra 23 (1995), no. 5, 1867-1897.

[Da] Davis, Michael W., Groups generated by reflections and aspherical manifolds not covered by Euclidean space, Ann. of Math., v.117(2), 1983, 293-324.

[ER] P. Etingof, E. Rains, New deformations of group algebras of Coxeter groups, Int. Math. Res. Not. 2005, no. 10, 635-646, math.QA/0409261

[S] C. T. Simpson, Products of matrices, Differential geometry, global analysis, and topology (Halifax, NS, 1990), 157-185, CMS Conf. Proc., 12, Amer. Math. Soc., Providence, RI, 1991.

[Yu] S. Yuzvinsky, Linear representations of posets, their cohomology and a bilinear form. European J. Combin. 2 (1981), no. 4, 385-397.

[Ma] The Magma website, http://magma.maths.usyd.edu.au

E-mail address: etingof@math.mit.edu

E-mail address: rains@math.ucdavis.edu 